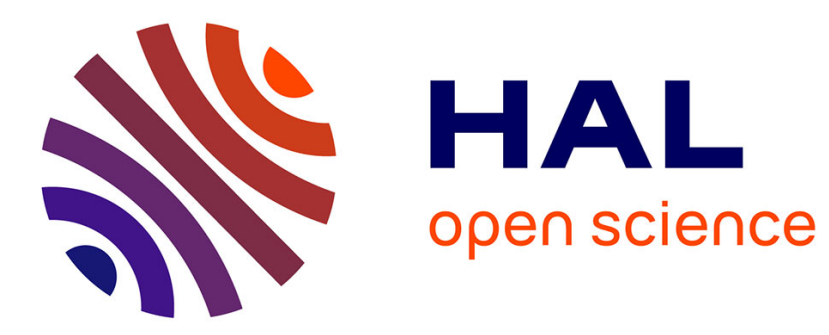

\title{
Original Broadcasting Technique in Radio Networks
}

Benoit Darties

\section{To cite this version:}

Benoit Darties. Original Broadcasting Technique in Radio Networks. CoNEXT '05: Conference on Emerging Network Experiment and Technology, 2005, Toulouse, France. pp.238-239, 10.1145/1095921.1095959 . lirmm-00177931

\section{HAL Id: lirmm-00177931 https://hal-lirmm.ccsd.cnrs.fr/lirmm-00177931}

Submitted on 12 Oct 2007

HAL is a multi-disciplinary open access archive for the deposit and dissemination of scientific research documents, whether they are published or not. The documents may come from teaching and research institutions in France or abroad, or from public or private research centers.
L'archive ouverte pluridisciplinaire HAL, est destinée au dépôt et à la diffusion de documents scientifiques de niveau recherche, publiés ou non, émanant des établissements d'enseignement et de recherche français ou étrangers, des laboratoires publics ou privés. 


\title{
Original Broadcasting Technique In Radio Networks
}

\author{
Benoît Darties
}

\begin{abstract}
This article presents a original tree-based broadcasting technique in known-topology radio networks. The main advantage of our strategy is it can be performed in asynchronous communication models using a protocol without acknowledgment. Considering this strategy, we introduce introduce first results for the problem of finding a broadcasting solution which minimizes the cost in time and/or energy.

Keywords-radio network, broadcast, tree-based strategy
\end{abstract}

\section{INTRODUCTION}

The growing interest in wireless communication networks provides a considerable attention to the radio networks. One main feature of radio networks is that a node have to receive a message from only one adjacent node at a given time. If not, the transmission is scrambled and the incoming message may be compromised. Moreover, in the most common networks, a single emission propagates in all direction, so can be received by the entire neighborhood of the emitter. Contrary to wired networks, there is no collision detection protocol. None of the nodes can determine by itself if the message has been received correctly by its recipients.

Broadcasting a message from a single source node is a common operation used in a lot of routing protocols. Because of those characteristics, broadcasting in radio networks becomes a difficult task: flooding techniques, which are successfully employed in wired networks, generate here lots of scrambled transmissions. Then we require the use of complex protocols with acknowledgments to ensure the message has been correctly delivered: this maintains interferences. Minimizing the time and/or the energy consumed by emissions during a broadcast has received a considerable attention in previous works. The configuration which considers a synchronous communication model, where the nodes are allowed to emit several times and temporize between their emissions, and where the global topology is known by a supervisor, has been particularly studied. Considering this one, finding a schedule which minimizes broadcast time is a NP-Complete problem[1]. A deterministic algorithm in [2] builds a broadcast using $O\left(D \log ^{2} n\right)$ steps, where $D$ is the graph eccentricity. In [3], 2-diameter graphs requiring $\Omega\left(\log ^{2} n\right)$ steps are mentioned. A randomized algorithm with $O\left(D \log n+\log ^{2} n\right)$ rounds was also proposed in [4]. The best known upper bound is $O\left(D+\log ^{5} n\right)$ in [5]. This configuration was also studied when the topology of the network is unknown: Chlebus and al. proposed an upper bound of $O\left(n^{11 / 6}\right)$, in [6]. Other results gradually decrease this upper bound to $O\left(n^{5 / 3} \log ^{1 / 3} n\right)$ in [7], to $O\left(n^{3 / 2} \sqrt{(} \log n\right)$ in [8], to $\mathrm{O}\left(n^{3 / 2}\right)$ [9], and to $O(n \log n)$ in [10]. A lower bound of $\omega(n \log D)$ was also proposed in [12].

We consider in our work that the topology of the network is

Benoît Darties is a 1st Year PhD student at the LIRMM, University of Montpellier II, France. E-mail: benoit.darties@lirmm.fr. known by a supervisor. We propose then a new broadcasting strategy using a protocol without acknowledgment, and which can be applied as well in a in a synchronous than in a asynchronous model. We first describe the specificities of our strategy, then announce some results relatives to the availability of a such strategy and the minimization of cost in time in general graphs.

\section{Model Description}

The radio network is modelized by an undirected graph $G=$ $(V, E)$, where the vertices represent the nodes, and there is an edge between two nodes if and only if they are in the range of each other. In the followings, we note source node the single node which owns the message to broadcast. Transmitters are the nodes which have to relay the message to their neighborhood, receivers only receive it. A node receives correctly a message if only one transmitter from its neighborhood emits at a given time. Two communication models are considered:

- In a synchronous model, time is divided into equal length transmission slots. Each transmission occurs in a single slot; a node retrieving a transmission at a given step is ready to retransmit it as of the next step.

- In a asynchronous model, each node has an undetermined and unbounded delay of processing. When a node receives a message, it cannot retransmit it before it has processed it. Thus, we cannot divide time into equal length slots, since we do not know when a node will retransmit a message. We consider that an independent transmission can only scramble a fixed amount of consecutive transmissions. ${ }^{1}$

The strategy we consider to ensure a broadcast from a single node is described as follow: The message propagation in the network is controlled by assigning to each transmitter node a father node from which it can only retrieves the message. For a given transmitter node, emissions not coming from the father node are ignored, even if they are anterior to the father emission. Each transmitter node can transmit a message only once, and cannot temporize between the reception and the emission. Eg. in a synchronous model, a node which receives the message from its father at step $k$ must retransmit it at step $k+1$. In a asynchronous model, if the sender is the father node, a node retransmits the message immediately after having proceeded it. The structure describing the paternity relations between transmitter nodes is a rooted tree where the source node is the root, vertices are the transmitter nodes, and an edge exists between a node and its father. This dominating tree ${ }^{2}$ describes under some conditions a broadcasting solution with our strategy. The depth of the tree is proportional to the required time to broadcast, and the num-

\footnotetext{
${ }^{1}$ eg. if delays of transmission are equal for each pair of nodes, then an independent transmission cannot scramble more than two consecutive transmissions.

2 a subgraph $G^{\prime}$ from $G$ which is a tree, and where each vertex in $G$ in included in $G^{\prime}$, or adjacent to a vertex in $G^{\prime}$
} 
ber of node to the energy consumed by emissions. Using this strategy requires a very simple protocol (each emitter just has to add its identity to the headers of the message it sends) and really differs from a simple bipartition in transmitter-receiver nodes or from a common schedule.

\section{MAIN RESUlTS}

We propose a lower bound for the number of transmitters. For any broadcast on a $n$-nodes network, we need $|T| \geq \frac{n-2}{\Delta-1}$ where $T$ is the set of transmitters, and $\Delta$ the maximum degree of $G$.

\section{A. in a synchronous model}

Our first results consider the tree-based strategy in a synchronous model. The message propagation describes here a particular dominating tree rooted on the source node: for a transmitter node $t$ at depth $d(t)$ in the tree, its father must be at depth $d(t)$ 1 , and no other adjacent transmitter must be at depth $d(t)-1$, to avoid interferences. Each receiver node $v$ must have at least one adjacent transmitter with a depth different from all the other ones. Every dominating tree which agrees with the precedings describes a valid broadcast solution. A tree resulting from a Depth First Search owns theses properties, then justify the existence of a solution for each instance. Our research gives then the followings results :

Theorem 1: Finding the tree which minimizes the time of broadcasting in a synchronous model is a NP-Hard and no-APX problem.

Proof: We consider a graph $G_{c}=\left(V_{c}, E_{c}\right)$ from the Minimum Vertex Coloration problem. We build a graph $G=(V, E)$ as follow: we copy each vertex $x_{i} \in V_{c}$ in $V$ and form a clique with them. For each $x_{i} \in V$, we add an adjacent vertex $x_{i}^{\prime}$ in $V$. For each $\{i, j\} \in E_{c}$, we add a vertex $x_{\{i, j\}}$ in $V$, and the edges $\left\{x_{i}, x_{\{i, j\}}\right\}$ and $\left\{x_{j}, x_{\{i, j\}}\right\}$ in $E$. We add a source node linked to all $x_{i}$. Each vertex $x_{i}^{\prime}$ ensure that $x_{i}$ have to transmit. Each vertex $x_{\{i, j\}}$ prevents $x_{i}$ and $x_{j}$ to transmit during the same step. When the source node emits, all vertices $x_{i}$ receive the message. Finding a dominating tree in $G$ which minimizes the time of broadcasting in $k+1$ steps give also a minimal coloration in $G_{c}$ with $k$ colors, by affecting the same color to the vertices $x_{i} \in V$ at the same depth in the tree. The reciprocal stands. Our reduction the preserves the approximability ratio of Minimum Vertex Coloration, which is an NP-Hard and no-APX problem. We conclude our problem is NP-Hard and no-APX on general graphs.

\section{B. in a asynchronous model}

When we consider our tree-based strategy in a asynchronous model, a broadcast can be defined as a particular dominating tree, like in he synchronous model. But we do not know when the different emissions occur. Then for a given transmitter node, no other adjacent transmitter must transmit at the same time as its father. The analysis of the situation results in if two transmitters are adjacent in the graph, then they must be on the same branch on the tree, or have the same father. For a receiver node, a certain percentage of its adjacent transmitters has to be on the same branch on the tree. ${ }^{3}$ A Depth First Search gives a such

\footnotetext{
${ }^{3}$ this percentage depends of the conditions on the asynchronous model. Eg. if transmissions delays are equals for each pair of nodes, for a given node at least $2 k / 3+1$ adjacent transmitters have to be on the same branch.
}

tree, in which for a given receiver node, all adjacent transmitters are on the same branch. We note the following result :

Theorem 2: Finding the tree which minimizes the time of broadcasting is a NP-Hard problem.

Proof: We use a common reduction to Exact-Set-Cover. From an instance $I$ of Exact Set Cover, we build the bipartite graph $G=(X, Y, E)$ as follows. For each set $S_{i}$ we add a vertex $x_{i}$ in $X$. For each element $e_{i}$ we add a vertex $y_{i}$ in $Y$. We add a edge $\left(x_{i}, y_{j}\right)$ iff $e_{j} \in S_{i}$. We add a source node $s$ linked to each $x_{i}$. If there exists a dominating tree with depth equals to 2 , which minimizes the time of broadcasting, then we can solve the instance $I$ of Exact-Set-Cover, and reciprocally.

\section{CONCLUSION}

We have shown our tree-based strategy represents an original alternative for the broadcast problem in wireless networks, which work as well in synchronous as in asynchronous communication models. Optimizing the time of broadcast with this strategy is a NP-complete and no-APX problem for general graphs. The goal of our work is to find good heuristics for building a tree with good performances in term of time and energy. Only a few results are presented here, more are mentioned in [13], and deals with specific topologies like grids, or tori. Other perspectives consider unit disk graph topologies, for their ability to represent effectively radio networks.

\section{REFERENCES}

[1] I. Chlamtac and S. Kutten, "On broadcasting in radio networks - Problem analysis and protocol design," IEEE Transactions on Communications, vol. 33, pp. 1240-1246, Dec. 1985.

[2] I. Chlamtac and O. Weinstein, "The wave expansion approach to broadcasting in multihop radio network," IEEE Transaction Communication, , no. 39, pp. 426-433, 1991.

[3] N. Alon, A. Bar-Noy, N. Linial, and D. Peleg, "A lower bound for radio broadcast,” J. Comput. Syst. Sci., vol. 43, no. 2, pp. 290-298, 1991.

[4] R. Bar-Yehuda, O. Goldreich, and A. Itai, "On the time-complexity of broadcast in multi-hop radio networks: An exponential gap between determinism and randomization.," J. Comput. Syst. Sci., vol. 45, no. 1, pp. 104-126, 1992.

[5] Iris Gaber and Yishay Mansour, "Broadcast in radio networks," in SODA '95: Proceedings of the sixth annual ACM-SIAM symposium on Discrete algorithms, Philadelphia, PA, USA, 1995, pp. 577-585, Society for Industrial and Applied Mathematics.

[6] B. Chlebus, L. Gạsieniec, A. Gibbons, A. Pelc, and W. Rytter, "Deterministic broadcasting in unknown radio networks," in SODA '00: Proceedings of the eleventh annual ACM-SIAM symposium on Discrete algorithms, Philadelphia, PA, USA, 2000, pp. 861-870, Society for Industrial and Applied Mathematics.

[7] G. De Marco and A. Pelc, "Faster broadcasting in unknown radio networks," Inf. Process. Lett., vol. 79, no. 2, pp. 53-56, 2001.

[8] D. Peleg, "Deterministic radio broadcast with no topological knowledge," 2000.

[9] B. Chlebus, L. Gạsieniec, Anna Östlin, and J.M. Robson, "Deterministic radio broadcasting," in ICALP '00: Proceedings of the 27th International Colloquium on Automata, Languages and Programming, London, UK, 2000, pp. 717-728, Springer-Verlag.

[10] M. Chrobak, L. Gasieniec, and W. Rytter, "Fast broadcasting and gossiping in radio networks," J. Algorithms, vol. 43, no. 2, pp. 177-189, 2002.

[11] D. R. Kowalski and A. Pelc, "Faster deterministic broadcasting in ad hoc radio networks," SIAM J. Discret. Math., vol. 18, no. 2, pp. 332-346, 2005.

[12] A. Clementi, A. Monti, and R. Silvestri, "Distributed broadcast in radio networks of unknown topology," Theor. Comput. Sci., vol. 302, no. 1-3, pp. 337-364, 2003.

[13] B. Darties, "Diffusion dans les rseaux sans fil longueur d'onde unique," Tech. Rep. 11856, LIRMM, 161, rue Ada, 34090 Montpellier, Janvier 2005 . 